\title{
Balanoposthitis associated with Gardnerella vaginalis infection in men
}

\author{
G R KINGHORN, * B M JONES, † F H CHOWDHURY,* AND I GEARY† \\ From the *Department of Genitourinary Medicine, Royal Infirmary, and the +Department of Medical \\ Microbiology, University of Sheffield Medical School, Sheffield
}

SUMMARY Fourteen of $194(7 \cdot 2 \%)$ consecutive unselected men had positive culture results from genital swabs for Gardnerella vaginalis. A higher yield of isolates was obtained from preputial $(93 \%)$ than from urethral swabs $(64 \%)$. Of the 14 men, two had no detectable genital abnormality, eight non-gonococcal urethritis, and nine balanoposthitis. The urethral isolation rates for $G$ vaginalis in men with and without non-gonococcal urethritis were not significantly different, but preputial isolation rates were significantly higher $(\mathrm{P}<0.001)$ in men with balanoposthitis than in those without. The prevalence rate for $G$ vaginalis in men with non-candidal balanoposthitis was $31 \%$.

In a second study, concomitant Bacteroides species were isolated in preputial swabs from nine of $12(75 \%)$ men with $G$ vaginalis-associated balanoposthitis and may play a role in its pathogenesis.

\section{Introduction}

Gardnerella vaginalis (Haemophilus vaginalis, Corynebacterium vaginale) has been described as the most prevalent sexually transmitted organism in the causation of bacterial vaginitis. ${ }^{1}$ Although women may harbour the organism without symptoms (as is the case with almost all genital pathogens), many infected women complain of an excessive malodorous vaginal discharge which is relieved when the organism is eradicated.

Evidence for the sexual transmission of $G$ vaginalis rests on the higher prevalence rates in the more sexually active women attending sexually transmitted disease (STD) clinics ${ }^{2}$ compared with those attending family planning ${ }^{3}$ or gynaecological clinics and on the high isolation rates from the urethra ${ }^{4-6}$ or urine ${ }^{7}$ of men known to be consorts of women harbouring $G$ vaginalis. Although $G$ vaginalis has been isolated in men with non-gonococcal urethritis (NGU), ${ }^{89}$ a causal role for the organism has not been established and asymptomatic colonisation of the male genitalia is assumed to be the rule. ${ }^{10}$

Previous work in this department ${ }^{2}$ showed that $G$ vaginalis was isolated from urethral, urine, or

Address for reprints: $\operatorname{Dr}$ G $R$ Kinghorn, Department of Genitourinary Medicine, Royal Infirmary, Sheffield S6 3DA

Accepted for publication 19 November 1981 prostatic specimens in only a few men who were consorts of women infected with $G$ vaginalis. In two men, however, much heavier growths of the organism were obtained from preputial swabs than from concomitant urethral swabs.

In this study we have investigated further the prevalence of $G$ vaginalis in urethral and preputial swabs from a group of consecutive unselected men and attempted to determine whether the presence of $G$ vaginalis on the male genitalia is associated with signs or symptoms of disease.

\section{Patients and methods}

One hundred and ninety-four consecutive unselected men presenting to the department of genitourinary medicine at this hospital with new complaints were prospectively studied at their first attendance. Urethral and preputial swabs (in circumcised men swabs were rolled on the glans penis and the coronal sulcus) were taken routinely, each immediately placed in peptone starch dextrose (PSD) transport medium, and left at room temperature until sent to the laboratory. This was usually done within an hour; the longest delay in transportation was three hours.

\section{CULTURAL DIAGNOSIS}

Swabs were inoculated on to Columbia blood agar plates $^{2}$ and incubated in $5 \% \mathrm{CO}_{2}$ at $37^{\circ} \mathrm{C}$. After 48 
hours $G$ vaginalis appeared as round raised glistening colonies, $0.5 \mathrm{~mm}$ in diameter, and often with a narrow zone of $\alpha$-haemolysis. Gram-stained films of these colonies showed slender Gram-variable bacilli. Pure subcultures were confirmed by their inhibition by hydrogen peroxide, a negative catalase test result, and fermentation of glucose, maltose, starch, but not mannitol.

\section{STATISTICAL ANALYSIS}

The sites of isolation of $G$ vaginalis and the clinical presentations in men with $G$ vaginalis infections and in those without were compared by Yates's modified $\chi^{2}$ test.

\section{SECONDARY STUDY}

In a second study we investigated symptomatic men and women with culture-confirmed $G$ vaginalis infection for the presence of concomitant anaerobic bacteria using the cultural methods of Duerden et $a l .{ }^{11}$ The swabs taken for $G$ vaginalis isolations were used for this.

\section{Results}

\section{ISOLATION RATE}

Of the 194 men in the initial prospective study, $G$ vaginalis was isolated from the urethral or preputial swabs or both in 14 , an isolation rate of $7 \cdot 2 \%$.

\section{SITES OF ISOLATION}

All 14 men were uncircumcised. The preputial swabs were the more productive; $13 / 14(93 \%)$ of the preputial swabs gave positive results compared with nine $(64 \%)$ of the urethral swabs.

\section{CONCOMITANT GENITAL CONDITIONS}

Only two men were without symptoms or signs of genital infection. Eight men had non-gonococcal urethritis, nine balanoposthitis, and one each genital herpes, genital warts, and pediculosis pubis.

Of 80 men presenting with NGU, $G$ vaginalis was isolated from the urethra of six compared with three $(2 \cdot 5 \%)$ of 114 without NGU $\left(\chi_{1}^{2}=1 \cdot 5, \mathrm{P}>0 \cdot 2\right)$.

Of 39 men with balanoposthitis, $G$ vaginalis was isolated from the prepuce of nine compared with four of 155 men without balanoposthitis $\left(\chi_{1}^{2}=17 \cdot 8\right.$, $\mathrm{P}<0.001)$. Yeasts were isolated from preputial swabs of 10 men with balanoposthitis, but in no patient were yeasts and $G$ vaginalis isolated together. Thus, nine of $29(31 \%)$ men with non-candidal balanoposthitis had $G$ vaginalis isolated from their preputial swabs. No significant differences were found between men with balanoposthitis, with or without $G$ vaginalis infections, and those without balano- posthitis in the prevalence of preputial isolations of $\frac{\Phi}{\square}$ commensal organisms such as Staphylococcus albus, 는 diphtheroids, micrococci, or potential pathogens $\frac{}{\omega}$ such as Enterobacteriaceae, Staphylococcus aureus, or streptococci.

CONCOMITANT ANAEROBIC INFECTIONS In our second study, both $G$ vaginalis and Bacteroides $\overline{\text { क }}$ species were isolated from the same swabs from nine $\mathbb{\Phi}$ of $12(75 \%)$ men with $G$ vaginalis-associated balanoposthitis and from $22(73 \%)$ of 30 women with $G$ vaginalis-associated vaginitis. Such anaerobic. infections may play a role in the pathogenesis of $G \vec{\overrightarrow{ }}$ vaginalis-associated conditions.

\section{$G$ VAGINALIS-ASSOCIATED BALANOPOSTHITIS} Clinical features

A total of 21 men with balanoposthitis associated with the presence of $G$ vaginalis were seen in the two studies. The onset of symptoms occurred in all men 은 within seven days of their most recent sexual contact, most often within two days. They consisted of a mild $D$ irritation of the prepuce and glans penis usually? associated with an excessive of fensive-smelling sub- $\vec{\varphi}$ preputial discharge. Men with an acquired or congenital phimosis or long redundant prepuces in: whom hygiene was deficient were particularly affected. After retraction of the prepuce and removal of the mucoid discharge erythematous non-erosive macules or papules were visible on the mucosal aspect of the prepuce and the glans penis.

\section{Discussion}

Our prevalence rate of $7 \cdot 2 \%$ for $G$ vaginalis isolation $\stackrel{9}{=}$ in unselected men was similar to that reported by Karpovaskaya $^{13}(8 \%)$. The study confirms an earlier impression $^{2}$ that in uncircumcised men $G$ vaginalis $\delta$ more frequently colonises, or is easier to isolate $₹$ from, the glans penis and prepuce than from the $ᄋ$ urethra, where the organism can easily be eradicated by micturition. We found no evidence to implicate the organism as a cause of non-gonococcal urethritis, but our results suggest that subpreputial carriage of $\%$ $G$ vaginalis is associated with balanoposthitis in some $N$ men, particularly those with a relative phimosis. ్ㅓㅇ Typically, the condition is mild with preputial irrita- $O$ tion and an offensive fishy odour and is associated with signs of increased subpreputial mucoid dis- $\frac{\mathscr{D}}{\Phi}$ charge and mild inflammation of the glans penis and $\stackrel{\infty}{?}$ prepuce.

Further study is necessary to determine the pathogenesis of this condition. Chen et al ${ }^{13}$ have $\stackrel{\mathbb{Q}}{\stackrel{D}{\circ}}$ postulated that in non-specific vaginitis a symbiotic $\frac{}{\circ}$ relationship between $G$ vaginalis and other 
organisms, particularly anaerobic bacteria, results in the production of amines which produce the characteristic symptoms. The frequent association of $G$ vaginalis with Bacteroides species in subpreputial swabs from men with balanoposthitis suggests that a similar mechanism might result in the typical fishy smell and increased subpreputial secretion.

We thank Mr W E Shaw and his nursing staff for their generous co-operation with this study, and also Mrs C Treweek and Mrs E Welch for typing the manuscript.

\section{References}

1. Dunkelburg WE. Corynebacterium vaginale. A review. Sex Transm Dis 1977; 4:69-75.

2. Bhattacharyya MN, Jones BM. Haemophilus vaginalis infection-its diagnosis and treatment. J Reprod Med 1980;24:71-5.
3. Bramley HM, Dixon RA, Jones BM. Haemophilus vaginalis (Corynebacterium vaginale; Gardnerella vaginalis) in a family planning clinic population. Br J Vener Dis 1981;57:62-6.

4. Gardner $\mathrm{HL}$, Dukes $\mathrm{CD}$. Haemophilus vaginalis vaginitis. Am J Obstet Gynecol 1955;69:962-76.

5. De La Fuente F, Rico LR, Soria F. Hemofilasis Urogenital Afection Venerea? Rev Esp Obstet Ginecol 1959;18:252-4.

6. Pheifer TA, Forsyth PS, Durfee MA, Pollock HM, Holmes KK. Non-specific vaginitis. Role of Haemophilus vaginalis and treatment with metronidazole. N Engl J Med 1978;298: 1429-33.

7. Leopold $\mathrm{S}$. Heretofore undescribed organism isolated from the genito-urinary system. US Armed Forces Medical Journal 1953;4:263-6.

8. Dunkelberg WE, Woolvin SC. Haemophilus vaginalis relative to gonorrhoea and male urethritis. Milit Med 1963;128: 1098-101.

9. Sylvestre L, Ethier J. Treatment of urethritis in males with oxytetracycline. Can Med Assoc J 1963;89:1218-20.

10. Gardner HL. Haemophilus vaginalis after 25 years. Am J Obstet Gynecol 1980; 137:385-91.

11. Duerden BI, Holdbrook WP, Collee JG, Watt B. The characterisation of clinically important Gram-negative anaerobic bacilli by conventional bacteriological tests. J Appl Bacteriol 1976; 40: 163-88.

12. Karpovaskaya $O$. The role of Haemophilus vaginalis infection of the female genitalia. Akush Ginekol (Mosk) 1971;47:33.

13. Chen KCS, Forsyth PS, Buchanan TM, Holmes KK. Amine content of vaginal fluid from untreated and treated patients with non-specific vaginitis. J Clin Invest 1979;63:828-35. 